\title{
A MACROPOLYCYCLE WITH TWO CYCLOHEXANE RINGS FUSED TO A 1,4-DIAZA-7-OXA-CYCLONONANE CORE
}

\author{
Geoffrey A. Lawrance, " Marcel Maeder and Mark J. Robertson \\ Discipline of Chemistry, School of Environmental and Life Sciences, The University of Newcastle, Callaghan, \\ New South Wales 2308, Australia
}

\begin{abstract}
Reaction of ethane-1,2-diamine with cyclohexene oxide produces the diaminodiol 1,2-bis(2'hydroxycyclohexylamino)ethane. This undergoes an Okahara ring closure to produce a new diaazaoxa macrocycle 2,5-diaza-12-oxa[12.4. $\left.0^{1,13} \cdot 0^{8,11}\right]$ tricycloseptadecane, consisting of three fused rings with a core cyclononane unit containing an $\mathrm{N}_{2} \mathrm{O}$ heteroatom donor set. The relatively rigid saturated molecule was characterized spectroscopically, and molecular mechanics analysis identified a number of possible conformers, some of which are suitable for binding metal ions due to appropriate heteroatom lone pair orientations. A palladium(II) complex bound through only the two $\mathrm{N}$ donors was prepared as additional characterization of the polyheterocycle isolated.
\end{abstract}

Introduction

Both aromatic and aliphatic macrocyclic heterocycles with nitrogen and oxygen donors have been synthesised as ligands for complexation of metal ions (1). The large aliphatic ring systems display a flexibility that means they are usually not preorganised for metal binding. Addition of further methylene 'straps' between nitrogen heteroatoms can enforce rigidity, but at the expense of altering steric and electronic effects arising from the conversion of secondary amines to tertiary amines (2). Alternatively, fusing small rings to the carbon framework of the macrocycle can increase bulk and rigidity while leaving the donor groups untouched. There have been a number of examples reported recently of both triaza (3) and tetraaza (4)-(6) macrocycles where at least one cyclohexane ring is fused to a macrocyclic ring containing heteroatoms, prepared by either pure organic or metal-directed synthetic routes. These heterocycles are, in principle, capable of binding metal ions and their application for this purpose is described in the reports that have appeared.

In an attempt to introduce greater rigidity and bulk into relatively small cyclononane macrocycles containing three heteroatoms, we have been examining strategies directed towards developing systems with two or three cyclohexane units fused to a cyclononane backbone that also incorporates three heteroatoms in the 1-,4- and 7-positions for metal binding. These systems not only promise more rigid environments but are inherently chiral, thus having potential as ligands in metal-promoted asymmetric synthesis. Here, we report the synthesis and characterization of the first $\mathrm{N}_{2} \mathrm{O}$-cyclononane with two fused cyclohexane rings. 


\section{Experimental}

1,2-Bis(2'-hydroxycyclohexylamino)ethane, 1: This was prepared from ethane-1,2-diamine dissolved in $\mathrm{E} \mathrm{tOH}$ and cyclohexene oxide, essentially as described elsewhere (7).

2,5-Diaza-12-oxa[12.4. $0^{1,13} \cdot 0^{6,11}$ ]tricycloseptadecane, 2: A mixture of $0.77 \mathrm{~g}(3 \mathrm{mmol})$ of 1 and $0.6 \mathrm{~g}$ (15 mmol) of $\mathrm{NaOH}$ was suspended in $100 \mathrm{~mL}$ of monoglyme under gentle heating and stirring. To this suspension was added dropwise a solution of $0.572 \mathrm{~g}$ ( $3 \mathrm{mmol})$ of freshly recrystallised toluenesulfonyl chloride in monogiyme $(30 \mathrm{~mL})$, with stirring. The resultant mixture was then heated to $60^{\circ} \mathrm{C}$ and kept at this temperature for $36 \mathrm{hrs}$. After cooling to RT, white solid precipitated was filtered off, leaving a clear solution that was then evaporated to dryness to leave a yellowish oil. Over a period of a week, off-white crystals formed and these were collected, and recrystallised from acetone to yield a white crystalline solid $[0.2 \mathrm{~g}$, 28\%]. NMR spectrum (DMSO) ${ }^{1} \mathrm{H}: \delta 1.0(\mathrm{~m}), 1.1-1.4(\mathrm{~m}), 1.5-2.1(\mathrm{~m}), 2.2-2.4(\mathrm{~m}, 2 \mathrm{H}), 2.6(\mathrm{q}), 2.8(\mathrm{q}) ;{ }^{13} \mathrm{C}$ ( ${ }^{\dagger} H$ decoupled): $\delta \square 24.1,28.0,28.1,33.8,37.9,40.6,41.3,42.9,50.3,64.1,67.176 .5$ ppm. IR spectrum (KBr disc): 3297, $3156\left(\mathrm{~N}-\mathrm{H}\right.$ ); $1063 \mathrm{~cm}^{-1}$ (C-O). GC-MS(El): (2-H) m/z 237 (calc. 237), (2-OH 220 (calc. 220), Microanalysis: $\mathrm{C}_{14} \mathrm{H}_{26} \mathrm{~N}_{2} \mathrm{O} 0.5 \mathrm{H}_{2} \mathrm{O}$ requires $\mathrm{C} 68.0, \mathrm{H} 11.0, \mathrm{~N} 11.3 \%$. Found $\mathrm{C} 68.5, \mathrm{H} 11.0, \mathrm{~N} 11.2 \%$. $\left[\mathrm{Pd}(2)\left(\mathrm{OH}_{2}\right)_{2}\right]\left[\mathrm{PdCl}_{4}\right] \cdot \mathrm{H}_{2} \mathrm{O}$ complex: Reaction of equimolar amounts of $2(0.037 \mathrm{~g}, 0.15 \mathrm{mmol})$ and $\mathrm{K}_{2}\left[\mathrm{PdCl}_{4}\right]$ $(0.05 \mathrm{~g}, 0.15 \mathrm{mmol})$ in water overnight yields the product as a brown solid, which was collected, washed with cold water and ethanol and air dried [0.055g, 57\%]. Microanalysis: $\mathrm{C}_{14} \mathrm{H}_{28} \mathrm{~N}_{2} \mathrm{O}_{4} \mathrm{Pd}_{2} \mathrm{Cl}_{4}$ requires $\mathrm{C} 26.15, \mathrm{H} 4.4$, N 4.35\%. Found: C 26.35, H 4.4, N 4.3\%.

\section{Results and Discussion}

The route used to form a new cyclononane ligand with two fused cyclohexane rings is shown below in Scheme 1. The reaction of the epoxide cyclohexene oxide on ethylene-1,2-diamine as reported (7) gives a diamine-diol compound 1 in high yield and purity. Ring opening occurs with exclusive formation of the trans configuration of the 1,2-disubstituted cyclohexane. The symmetry of this compound, 1 , is displayed by the simplicity of the ${ }^{13} \mathrm{C}$ NMR, consistent with the expectation of there being two magnetic equivalent halves about the $-\mathrm{H}_{2} \mathrm{C}-\mathrm{CH}_{2}$ - bond of the ethane-1,2-diamine residue. Intramolecular dehydration of this complex by an Okahara ring closure mechanism ( 8 ) then gives the cyclic ligand $\underline{2}$.

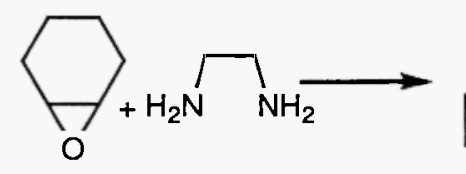

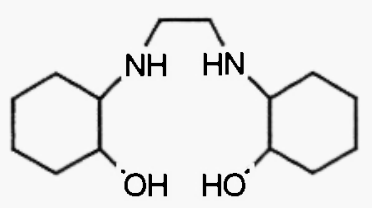

$\underline{1}$

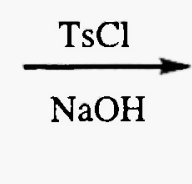

Scheme 1

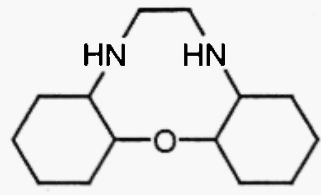

$\underline{2}$

The Okahara ring closure of 1 was completed using $\mathrm{NaOH}$ and tosyl chloride ( $\mathrm{TsCl}$ ) in monoglyme as the solvent. The crystallised yield of 2 was low $(-30 \%)$, but changing the reaction conditions as far as time and duration had no consistent effect, nor did using other metal hydroxides nor changing the solvent. The characterisation of the cyclic species $\underline{2}$ was completed using microanalysis, NMR (including DEPT) and 
GC/MS spectrometry. Ring formation in principle can lead to both meso and rac isomers, where the cyclohexane rings should be related by either a mirror plane or two-fold rotation axis. However, cyclisation may produce a conformationally rigid molecule where the two 'halves', equivalent in the precursor 1 , need no longer be symmetry-related, depending particularly on the orientation (exo or endo to the macrocyclic cavity) of the heteroatom lone pairs. Minimised structures, generated from molecular mechanics using a random kick process (9), illustrate this for $\underline{2}$ in Figure 1. It is notable that there exists magnetic inequivalence between carbon atoms of the cyclic molecule isolated, arising as a result of a 'frozen' macrocyclic ring framework. In the ${ }^{13} \mathrm{C}$ NMR spectrum, there are four methine carbon atoms, with the peaks at 76.5 and 67.1 ppm assigned to those adjacent to the oxygen atom, and the other two at 41.3 and 42.9 ppm resulting from the carbon atoms adjacent to the nitrogen atoms. The methylene fragments from ethylenediamine appear at 64.1 and $50.3 \mathrm{ppm}$, with other peaks upfield resulting from the methylene carbons within the cyclohexane framework. A lower symmetry structure such as that shown on the left in Figure 1 presumably occurs in the crystallised 2 isolated; while the crystallized product is a single isomer, the exact form must await crystal structure characterization.

The GC/MS results also provide further evidence that the cyclononane species was formed. The GC of the product of the Okahara ring closure reaction gave only the one peak. When this sample was spiked with a small amount of the precursor diol 1 and the GC experiment re-run with the same conditions, two peaks appeared, clearly indicative of 1 and 2 as different molecules. The molecular ion was also seen for the cyclic species 2 at $m / z 237$ amu. Further fragments, including one due to loss of the oxygen, were seen. There are, as expected, common fragments seen in the cyclic and acyclic compound mass spectra, which relates to an essentially common framework.
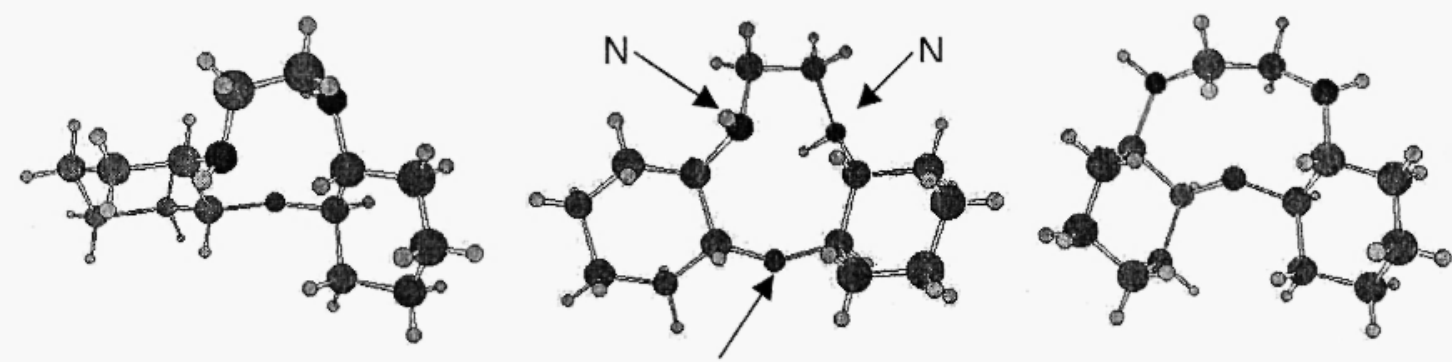

Figure 1. Examples of molecular mechanics minimised low energy rac and meso isomers of $\underline{2}$. Structures with different lone pair orientations on heteroatoms result from the minimization process employed; for example, lone pairs on $\mathrm{O}$ are exo (centre structure) and endo (right-hand structure) to the core cyclononane ring. Appropriate preorganisation of the heteroatom lone pairs is important for subsequent coordination to metal ions.

Reaction of 2 with $\left[\mathrm{PdCl}_{4}\right]^{2 \cdot}$ resulted in a complex being isolated as the $1: 1 \mathrm{M}: \mathrm{L}$ complex species $\left[\mathrm{Pd}(2)\left(\mathrm{OH}_{2}\right)_{2}\right]\left[\mathrm{PdCl}_{4}\right]$. With a square-planar cation, 2 can chelate only through two donor atoms, presumably nitrogen, with the third donor uncoordinated. These types of diamine chelate complexes are not unusual for palladium species, with the neutral ether oxygen atom not a preferred donor atom for metal ions. Since chelation to $\mathrm{Pd}$ (II) was achieved, appropriate preorganised endo,endo orientation of $\mathrm{N}$-donors in 2 is implied; 
together with NMR evidence, a structure similar to that on the left in Figure 1 satisfies the observations. The absence of an easily prepared 1:2 M:L species is notable. The effect of two cyclohexane rings on complexation in this instance may be quite substantial. Even if the lone pairs of the donor atoms are orientated endo to the ring in a direction applicable for metal coordination, as in not the case in all possible isomers, steric effects may now be important. The coordination chemistry of this and related molecules will be pursued independently.

\section{Conçlusion}

The formation of a sterically bulky polyheterocycle by the incorporation of extra fused cyclohexane rings has been achieved by an Okahara ring closure of a diol, itself formed from the ring opening reaction of an epoxide. Attempts to develop a similar system with three fused cyclohexane rings are in progress. The use of an ether type oxygen donor atom is not a preferred donor atom for many metal ions, and so introquction of a thioether via an episulfide reagent instead is planned. The inherent rigidity of this type of ligand may also mean that if the donor atoms are not oriented in the preferred direction for metal bonding. the resulting twisting of the hydrocarbon skeleton required to afford the right orientation may be an unfavourable high energy process; that is, the molecule must be appropriately predisposed, or organized, for coordination.

\section{Acknowledgement}

Support of this work by the Australian Research Grants Committee is gratefully acknowledged.

\section{References}

(1) D. Parker, 'Macrocycle Synthesis: a Practical Approach', Oxford University Press, 1996.

(2) T. N. Mali, P. W. Wade and R. D. Hancock, J. Chem. Soc., Dalton Trans., 67 (1992).

(3) S. W. Golding, T. W. Hambley, G. A. Lawrance, S. M. Luther, M. Maeder and P. Turner, J. Chem. Soc., Dalton Trans., 1975 (1999).

(4) I. Alfonso, C. Astorga, F. Rebolledo and V. Gotor, Tetrahedron: Asymmetry, 10, 2515 (1999).

(5) J. Cho, U. Lee and J. C. Kim, Trans. Met. Chem., 27, 429 (2002).

(6) P. V. Bernhardt, B. Elliott, G. A. Lawrance, M. Maeder, M. A. O'Leary, G. Wei and E. N. Wilkes, Aust. J. Chem., 47, 1771 (1994).

(7) A. S. de Sousa and R. D. Hancock, J. Chem. Soc., Chem. Commun., 415 (1995).

(8) P.-L. Kuo, M. Miki, I. Ikeda and M. Okahara, Tetrahedron Lett., 4273 (1978).

(9) P. Comba, T. W. Hambley, G. Lauer and N. Okon, 'MOMEC97, a molecular mechanics program for coordination compounds adapted to HyperChem', $2^{\text {nd }}$ edn, CVS, Heidelberg, 1997.

\section{Received on October 10, 2002.}

